\title{
Phylogenetic position of Diania challenged
}

ARISING FROM J. Liu et al. Nature 470, 526-530 (2011)

Liu et al. ${ }^{1}$ describe a new and remarkable fossil, Diania cactiformis. This animal apparently combined the soft trunk of lobopodians (a group including the extant velvet worms in addition to many Palaeozoic genera) with the jointed limbs that typify arthropods. They go on to promote Diania as the immediate sister group to the arthropods, and conjecture that sclerotized and jointed limbs may therefore have evolved before articulated trunk tergites in the immediate arthropod stem. The data published by Liu et al.1 do not unambiguously support these conclusions; rather, we believe that Diania probably belongs within an unresolved clade or paraphyletic grade of lobopodians.

Without taking issue with the interpretation of Diania offered by Liu et al. ${ }^{1}$, or of the manner in which they coded their characters, we were nonetheless unable to derive their cladogram optimally from the data published. Moreover, we could not replicate their results using any other plausible optimality criteria, or by varying additional parameters not specified by the authors.

Liu et al. ${ }^{1}$ report analysing their data in $\mathrm{PAUP}^{* 2}$ under maximum parsimony and with implied weights ${ }^{3}$ using $k=2$ (a rather arbitrary choice), but do not mention any other assumptions (for example, the imposition of character order). They obtained three most parsimonious trees, each of 130 steps. Straightforward replication of their stated settings yields 13 trees of just 90 steps each, the strict consensus of which is illustrated (Fig. 1). Why such a difference?

Several of their characters contained inapplicable or gap codings. These appear where a 'daughter' character is logically contingent upon the state of a 'parent', and cannot be coded when the parent is

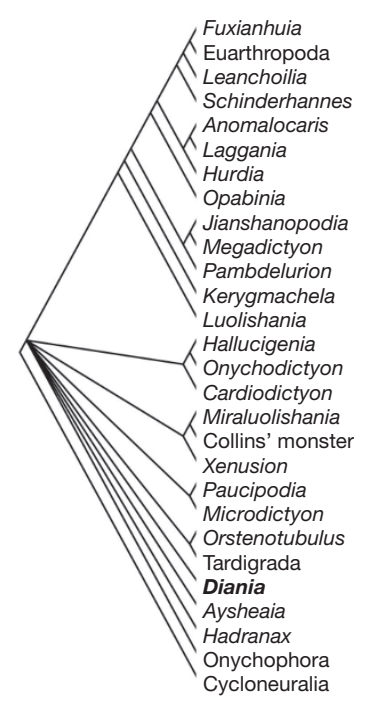

Figure $1 \mid$ The strict consensus of 13 most parsimonious trees $(L=90)$ obtained from the published data and settings specified by the authors. absent. For example, character 6 (position of frontal appendage) can only be coded in taxa that possess a frontal appendage (character 5) in the first instance (such that a " 0 " for character 5 necessitates a "-." for character 6). In morphological analyses such as this, inapplicable states are usually assumed to have no bearing on the analysis, being reconstructed passively in the light of known states. In analyses of nucleotide data, by contrast, gaps may alternatively be construed as a fifth and novel state, because shared deletions from some ancestral sequence may actually be informative. If this assumption is made with morphological data, however, all the logically uncodable states in a character are initially assumed to be homologous, and a legitimate basis for recognizing clades. At best, this assigns double weight a priori to absences in the 'parent' character (because the daughter is always contingent), and at worst is positively misleading. This is the approach that we believe Liu et al. ${ }^{1}$ may have taken. Reanalysis of their data using 'gapmode $=$ newstate' combined with 'collapse $=$ MinBrlen' settings in PAUP*2 produced some optimal trees of 130 steps. However, we were still unable to replicate the relationships shown in their Fig. 4, even when varying $k$ between 0 and 10 . Rather we either resolved Diania in a basal polytomy, or slightly higher in the tree but separated from the arthropods by at least five nodes.

At best, therefore, the position of Diania is highly labile and extremely sensitive to the precise methods used. We certainly feel that it is premature to draw conclusions regarding its supposedly pivotal position in the evolution of arthropods. However, our reanalyses do not challenge the more general conclusions of Liu et al. ${ }^{1}$ : namely that the full complement of arthropod characters were probably acquired piecemeal and possibly convergently. Many closely allied groups exploited successfully some but not all of the characters that typify the arthropod crown group. Only in retrospect do we discern a single, ladder-like trajectory through what was really a much more eccentrically branching bush.

Ross C. P. Mounce ${ }^{1}$ \& Matthew A. Wills ${ }^{1}$

${ }^{1}$ Department of Biology and Biochemistry, University of Bath, Bath BA2 7AY, UK.

e-mail: rcpm20@bath.ac.uk

Received 3 March; accepted 24 May 2011.

1. Liu, J. et al. An armoured Cambrian lobopodian from China with arthropod-like appendages. Nature 470, 526-530 (2011).

2. Swofford, D. L. PAUP*. Phylogenetic Analysis Using Parsimony (*and Other Methods) Version 4 (Sinauer Associates, 2002).

3. Goloboff, P. A. Estimating character weights during tree search. Cladistics 9, 83-91 (1993).

Author Contributions R.C.P.M. initiated this comment and reanalysed the data. M.A.W. highlighted the potential issue with gap codings. Both authors wrote the note.

Competing financial interests: declared none.

doi:10.1038/nature10266 


\section{Lobopodian phylogeny reanalysed}

ARISING FROM J. Liu et al. Nature 470, 526-530 (2011)

Liu et al. ${ }^{1}$ described an 'armoured' lobopodian, Diania cactiformis, from the Chengjiang Lagerstätte (China; Cambrian, stage 3); this fossil bears potentially arthropod-like articulated and possibly sclerotized appendages, but lacks a sclerotized body. A cladistic analysis resolved Diania as sister-taxon to arthropods. From this phylogenetic position the authors tentatively inferred that arthropodization (sclerotization of limbs) may have preceded arthrodization (sclerotization of body elements) in arthropod evolution. Although we concur with the reasoning behind this inference, it rests on a phylogenetic placement that our analysis of the published data set does not reproduce.

Our analyses were undertaken using implicit enumeration (branch and bound) in TNT (Tree Analysis using New Technology) v.1.1 (ref. 2), first repeating the analysis of Liu et al. ${ }^{1}$ with implied character weighting $(k=2)$ and additionally with equal character weighting; results are shown in Fig. 1b, c. In neither analysis was Diania resolved as sister-taxon to Arthropoda, instead belonging to an unresolved polytomy at the base of the resultant most-parsimonious trees. To confirm that the trees of Liu et al. ${ }^{1}$ are less parsimonious, a second pair of analyses was undertaken. Here tree topologies were constrained to the published strict-consensus topology (Fig. 1a), again using implied $(k=2)$ and equal weighting schemes. In both cases a single tree was found, substantially longer than those our first analyses recovered (16.4 as opposed to 12.4 steps under implied weighting; 119 as opposed to 89 steps under equal weighting). This demonstrates that the published topology is far from the most parsimonious that can be obtained from the data set. The use of a branch and bound (rather than heuristic) search indicates that this discrepancy is not a result of chance failure to hit upon the best solution; instead we believe that it reflects a methodological error in the original analysis.

The phylogeny recovered here suggests a polyphyletic origin of arthropodized limbs, where the arthropodization in Diania was acquired separately to that of the crown-group arthropods. It does not rule out the 'leg-first' (as opposed to arthrodization-first) scenario posited by Liu et al. ${ }^{1}$, but provides no support for it either. The recovered polychotomy demonstrates that the character coverage of Liu et al. ${ }^{1}$ is inadequate to resolve the interrelationships of lobopodians; a more comprehensive data set is required to assess the position and significance of Diania properly. We note, however, that our results from the Liu et al. ${ }^{1}$ data set (Fig. 1b) place the euarthropods within the dinocaridids (radiodonts and related taxa), and as such are similar to other analyses ${ }^{3,4}$ which have used more character- and taxon-rich data sets. The origin of arthropodized trunk limbs is problematical in this topology, as dinocaridids apparently lack any form of leg-like trunk appendage ${ }^{5}$. The increasingly detailed fossil record of stem-group euarthropods provides our best chance of resolving this issue, but as yet has failed to do so; unequivocal evidence for any particular ordering of acquisition in these characters is not yet available. Diania is a fascinating animal, but in its revised position it contributes little to this debate.

\section{David A. Legg ${ }^{1,2}$, Xiaoya $\mathrm{Ma}^{2}$, Joanna M. Wolfe ${ }^{3}$,}

Javier Ortega-Hernández ${ }^{4}$, Gregory D. Edgecombe ${ }^{2}$ \& Mark D. Sutton ${ }^{1}$

${ }^{1}$ Department of Earth Science and Engineering, Imperial College London, London SW7 2AZ UK.

e-mail: d.legg10@imperial.ac.uk a
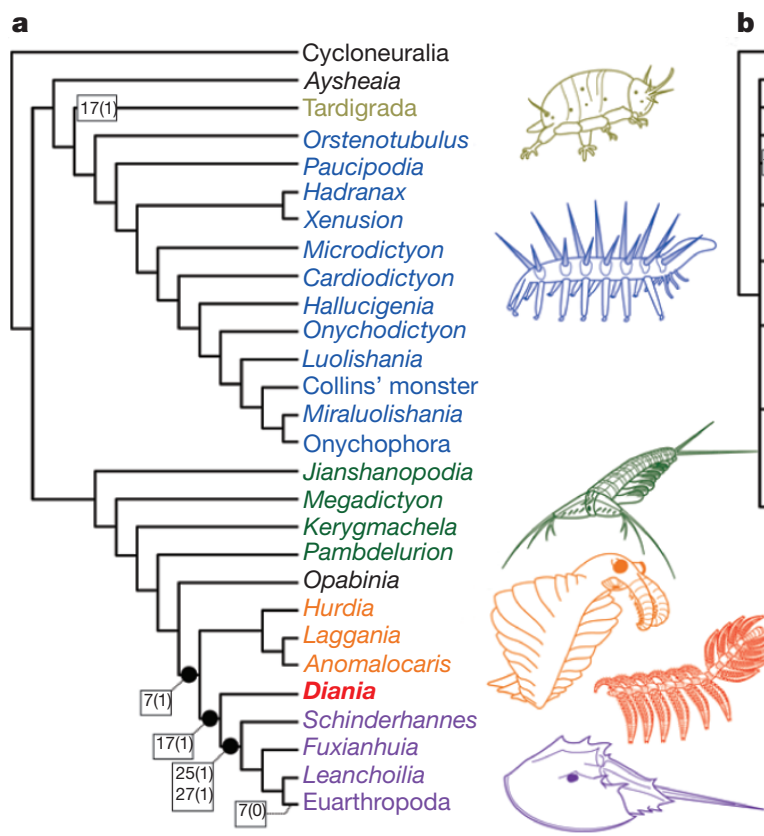

Figure $1 \mid$ Phylogeny of Cambian lobopodians and stem-group arthropods. a, Topology proposed by Liu et al. ${ }^{1}$ (implied weights, $k=2,16.4$ steps, consistency index $(\mathrm{CI})=0.336$, retention index $(\mathrm{RI})=0.546$; equal weights, 119 steps, $\mathrm{CI}=0.336, \mathrm{RI}=0.546)$. $\mathbf{b}$, Strict consensus of five most parsimonious trees using the methods of Liu et al. ${ }^{1}$ (implied weights, $k=2,12.4$ b

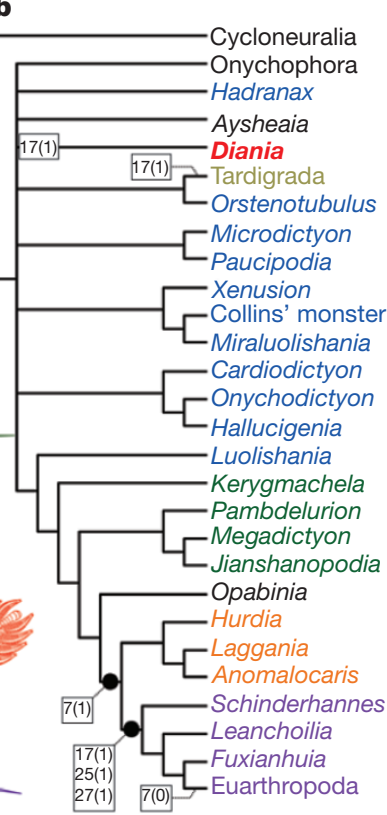

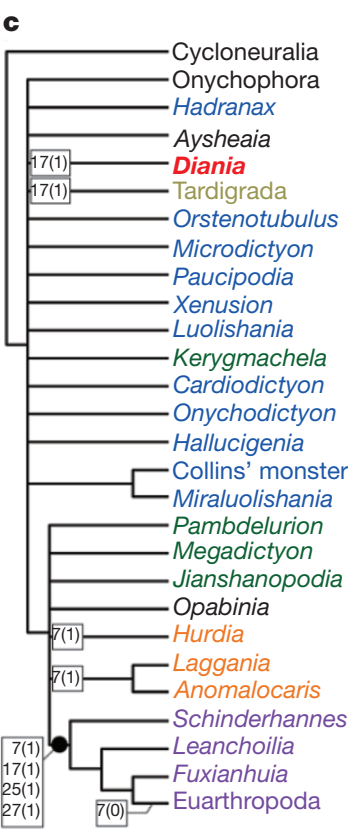

c

steps, $\mathrm{CI}=0.444, \mathrm{RI}=0.713)$. c, Strict consensus of 86 trees produced with equal character weighting ( 89 steps, $\mathrm{CI}=0.499, \mathrm{RI}=0.718$ ). Numbers represent important characters of Liu et al. ${ }^{1}$ relating to arthropodization and arthrodization. 
${ }^{2}$ Department of Palaeontology, Natural History Museum, London SW7 5BD, UK.

${ }^{3}$ Department of Geology and Geophysics, Yale University, New Haven, Connecticut 06520-8109, USA.

${ }^{4}$ Department of Earth Sciences, University of Cambridge, Cambridge CB2 3EQ, UK.

\section{Received 3 March; accepted 24 May 2011.}

1. Liu, J. et al. An armoured Cambrian lobopodian from China with arthropod-like appendages. Nature 470, 526-530 (2011).

2. Goloboff, P. et al. TNT, a free program for cladistic analysis. Cladistics $\mathbf{2 4 , 7 7 4 - 7 8 6}$ (2008).
3. Daley, A. C. et al. The Burgess Shale anomalocaridid Hurdia and its significance for early euarthropod evolution. Science 323, 1597-1600 (2009).

4. Ma, X. et al. Morphology of Luolishania longicruris (Lower Cambrian, Chengiiang Lagerstätte, SW China) and the phylogenetic relationships within lobopodians. Arth. Struct. Dev. 38, 271-291 (2009).

5. Zhang, X. \& Briggs, D. E. G. The nature and significance of the appendages of Opabinia from the Middle Cambrian Burgess Shale. Lethaia 40, 161-173 (2007).

Author Contributions: D.L. was responsible for writing and coordination of the manuscript; X.M., J.W., J.O.-H., G.E.D. and M.S. helped with discussion. D.L. and J.W. were also responsible for reanalysing the data. J-O.-H. produced the accompanying figure and M.S. aided with wording and stylistic editing of the original manuscript.

Competing financial interests: declared none.

doi:10.1038/nature10267

\section{Liu et al. reply}

REPLYING To R. C. P. Mounce \& M. Wills Nature 476, doi:10.1038/nature10266 (2011); D. A. Legg et al. Nature 476, doi:10.1038/nature10267 (2011);

We welcome the reanalyses by Mounce and Wills ${ }^{1}$ and Legg et al. ${ }^{2}$ of our paper ${ }^{3}$, and although we do not fully concur with their conclusions we are pleased that Diania has reopened the debate about key stages in arthropod evolution. We accept that the position of this fossil remains sensitive to parameters of analysis and in the original publication we conceded that our best-supported tree-Diania as sistergroup to (Schinderhannes + Euarthropoda) - could be subject to

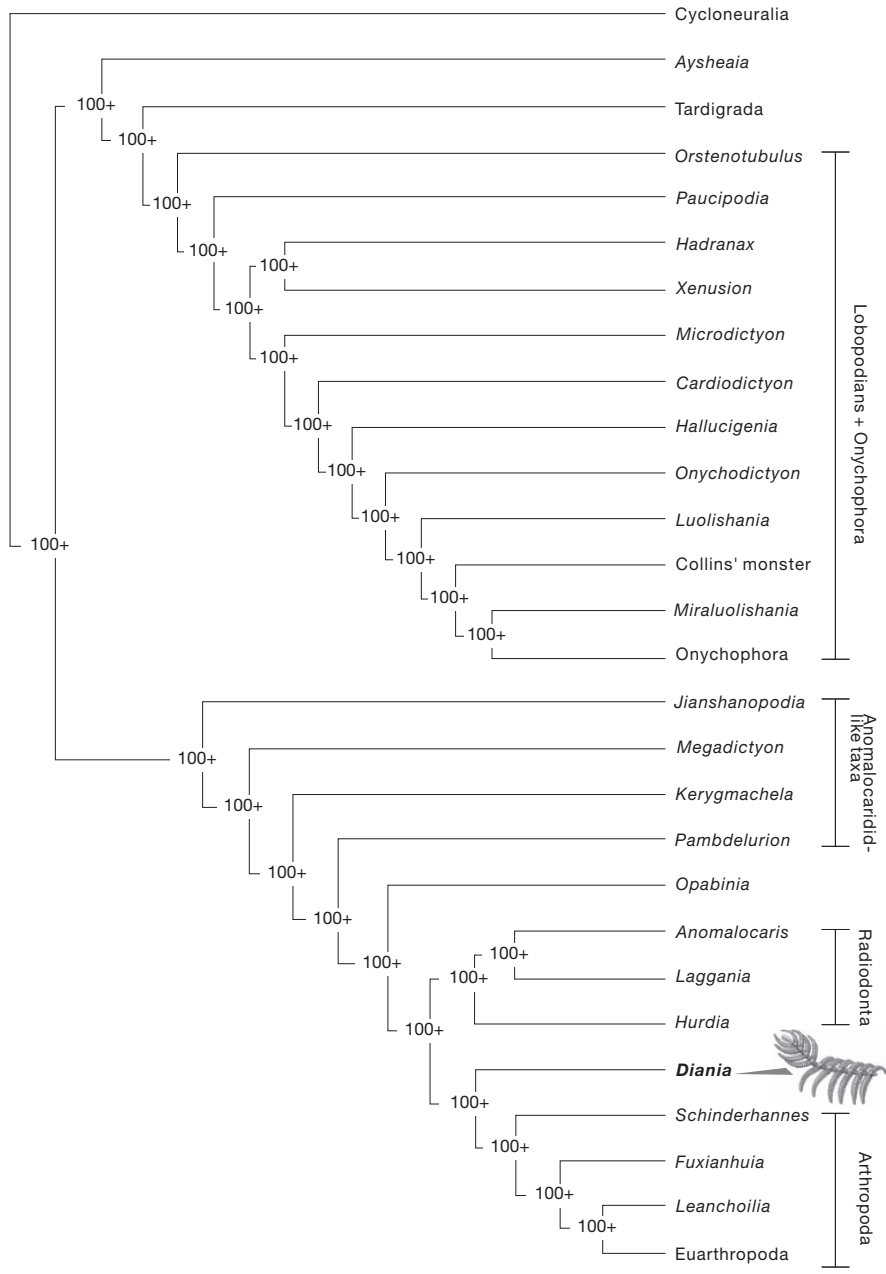

change, and that the 'walking cactus' may have a more basal position within the overall framework of the arthropod stem-group. These alternative treatments of our data would seem to confirm this suspicion, although we find the placement of Diania in an unresolved, and extremely basal, polytomy alongside velvet worms, tardigrades and various other lobopodians similarly problematical. We do not doubt that the authors' results ${ }^{1,2}$ are statistically well supported, but what do these cladograms tell us about the evolution of the group? Lobopodians are, by their nature, fairly simple and consequently yield few convincing synapomorphies, either with each other or with arthropods in general. As we discovered, this makes scoring a robust data matrix including both lobopodians and arthropods challenging, and we wonder whether the basal polytomies recovered here are simply due to clustering among taxa with few unequivocal apomorphies and/or much missing data.

Our original placement of Diania close to the euarthropods was strongly influenced by the character of jointed trunk appendages. We acknowledge an error in scoring characters 17 and 27 of Tardigrada, which should have been 0 and 1 , not 1 and 0 , respectively. We also accept the criticism by Mounce and Wills ${ }^{1}$ that parent-daughter characters are, to some extent, dependent upon one another and can artificially inflate support for particular clades. This situation is hard to avoid when selecting characters across a range of lobopod/arthropod fossils, which include taxa with unusual morphologies (for example, Opabinia) or where there are alternative hypotheses for the homology of a given lobopod feature and its probable arthropod equivalent: should a lobopod/dinocaridid flap be scored the same way as a euarthropod limb

Figure $1 \mid$ Reanalysis of our data using PAUP: bootstrap 50\% majority-rule consensus tree. Hierarchical structure in the data was assessed using the PTP test $^{6}$ as implemented in PAUP. Parsimonious trees were found through a heuristic search strategy using tree bisection-reconnection (TBR) branch swapping. In an initial analysis, all characters were treated as unordered. Branch support was based on calculating bootstrap values (10,000 replicates and with rearrangement limit of 10,000,000 rearrangements per additional sequence). A significant value in the PTP test $(P=0.01)$ does suggest the presence of a phylogenetic signal in the morphological data, supporting the given topology. Thirty-eight characters were parsimony-informative. The heuristic search for a maximum parsimony solution resulted in only one parsimonious tree (length $=121$, homoplasy index $(\mathrm{HI})=0.67$, consistency index $(\mathrm{CI})=0.33$, retention index $(\mathrm{RI})=0.55$, excluding 1 uninformative character). This tree contains 26 resolved nodes, of which many were strongly supported by bootstrap values $(100+)$ and in some nodes bootstrap values showed even greater resolution. 
exite; could both be subsumed under a 'biramous limb', etc.? Furthermore, a disadvantage of PAUP is that 'gaps' are automatically treated as missing in the analysis, and the only way to distinguish between them is to treat the gap as a new state; although a gap in our analysis refers to an inapplicable character, which is itself not a new state.

In our initial analysis data were entered into a matrix using MacClade version 3.05 (ref. 4). Analyses were performed with PAUP version $4.0 \mathrm{~b} 10$ (ref. 5), whereby multi-state characters were treated as 'unordered', other characters were treated as 'ordered', and a branch and bound search under implied weights $(k=2)$ produced many most parsimonious trees-including trees similar to those of Legg et al. ${ }^{2}$ and Mounce and Wills ${ }^{1}$. For the purpose of character state mapping, we selected one of these most parsimonious trees (see Fig. 4 in ref. 3 ) which we felt best reflected the complex relationships among lobopodians and arthropods. Also, a constrained analysis was conducted, and three monophyletic groups (lobopodians + Onychophora, Radiodonta, Arthropoda) were enforced. To verify the stability of this tree, we conducted bootstrap analysis and the result (Fig. 1) shows that the contained 26 resolved nodes of this tree were also strongly supported by bootstrap values $(100+)$ and in some nodes bootstrap values

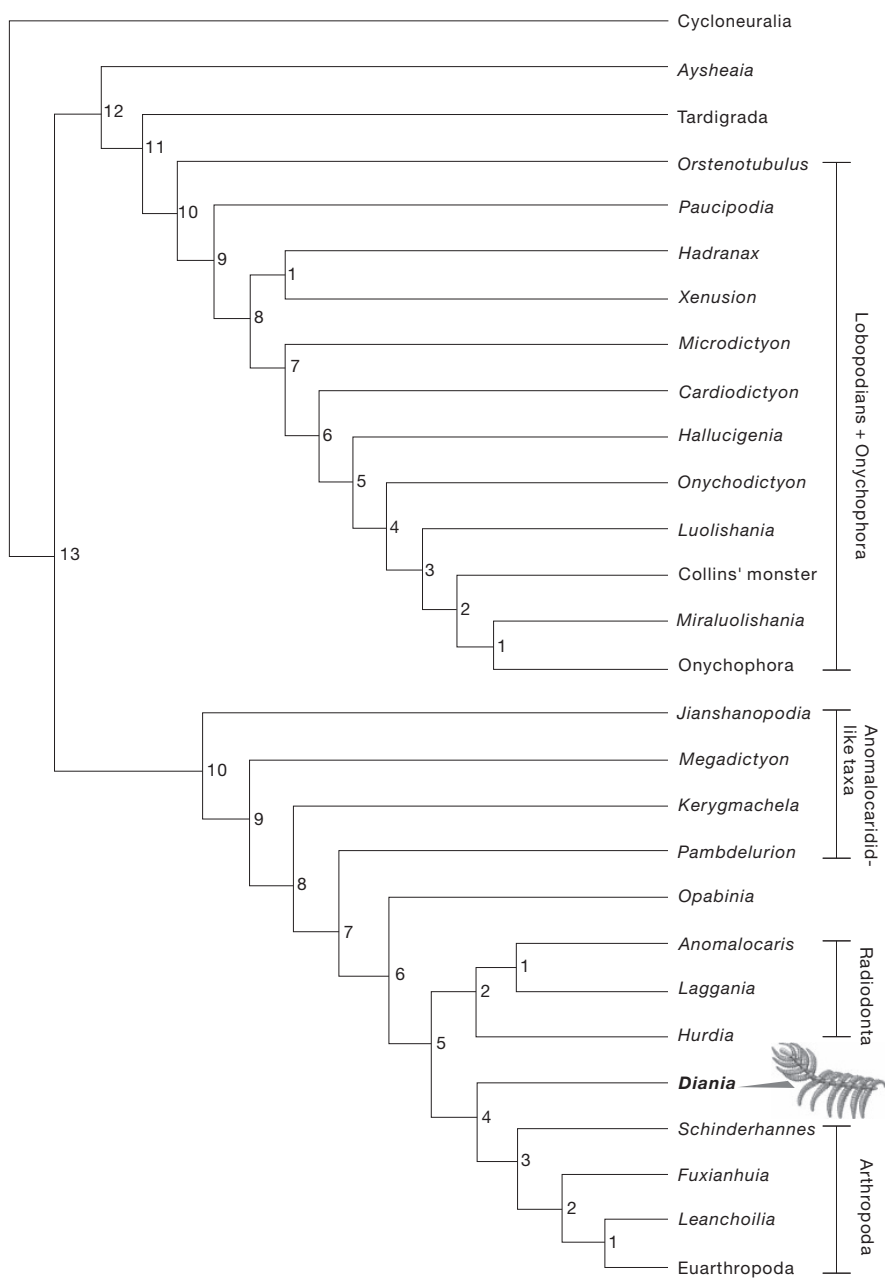

Figure $2 \mid$ Tree shows the Bremer support value, which strongly supports Diania being sister group to Arthropoda (Bremer index =4). Four additional steps would be required to disrupt the Diania-arthropod relationship. As we noted in the original description, Diania is the most arthropod-like of the lobopodians recorded so far-at least in terms of limb morphology - and we feel that it is better placed on the arthropod stem, rather than in an unresolved polytomy. showed more resolution. Additionally, a significant value of the partitioning tail permutation (PTP) test $(P=0.01)$ suggests the presence of a clear phylogenetic signal in the morphological data, also strongly supporting the topology shown. Furthermore, a test of Bremer support still supports our initial analysis (Fig. 2).

Legg et al. ${ }^{2}$ drew attention to the absence of sclerotized trunk limbs in dinocaridids (Anomalocaris, etc.), which remains for us one of the great puzzles of stem-group arthropod evolution. Put simply, dinocaridids have a more arthropod-like head region (cephalisation, eyes, sclerotized mouthparts), whereas Diania lacks such sophistication in the anterior body region, but has jointed trunk appendages. One solution to this puzzle would be to assume that dinocaridids also had trunk limbs, but lost them secondarily. In this scenario Diania could sit comfortably as sister group to a (Dinocaridida + Arthropoda) clade: all three sharing jointed appendages, the latter two sharing cephalisation, etc.

We accept that the tree of Legg et al. ${ }^{2}$ and Mounce and Wills ${ }^{1}$ is the strict consensus tree, but we feel that the strict tree is, in this case, too conservative to provide meaningful information about the early evolution of the arthropods. This was undoubtedly a complex process, and may have involved numerous parallel developments introducing homoplasy. Under these circumstances, the comb-like most parsimonious tree is unhelpful and exemplifies a disadvantage of parsimony in this instance when faced with a complex early radiation. Indeed, Legg et al. ${ }^{2}$ recognized that the strict consensus tree(s) "suggests a polyphyletic origin of arthropodized limbs". If true, this would be significant. In this context, Mounce and Wills ${ }^{1}$ seem to have overlooked the potential significance of their reanalysis of our data. Much evidence has been accumulated that Arthropoda is monophyletic, but the logical conclusion of both reanalyses is that jointed legs are homoplastic, at least within the lobopodian-arthropod assemblage. The implications of this are not trivial, bringing us close to the (largely discredited) Manton school of thought in which jointed appendages were proposed to have evolved in parallel in different (here euarthropod) lineages. At a fundamental level we need to know how easy it is to turn a soft, lobopodian limb into a sclerotized arthropod one, and whether this happened once, twice or on multiple occasions. For this reason alone, we believe that Diania is not merely fascinating, but remains invaluable to the evolutionary debate by challenging our notion of what it means to be an arthropod.

\section{Jianni Liu ${ }^{1,2}$, Michael Steiner ${ }^{2}$, Jason A. Dunlop ${ }^{3}$, Helmut Keupp ${ }^{2}$,} Degan Shu ${ }^{1,4}$, Qiang Ou ${ }^{4}$, Jian Han ${ }^{1}$, Zhifei Zhang ${ }^{1}$ \& Xingliang Zhang ${ }^{1}$

${ }^{1}$ Early Life Institute, State Key Laboratory of Continental Dynamics,

Department of Geology, Northwest University, Xi'an 710069, China.

${ }^{2}$ Department of Earth Science, Freie Universität Berlin, D-12249 Berlin, Germany.

e-mail: liu2009@zedat.fu-berlin.de or liujianni@126.com

${ }^{3}$ Museum für Naturkunde, Leibniz Institute for Research on Evolution and Biodiversity at the Humboldt University Berlin, D-10115 Berlin, Germany. ${ }^{4}$ School of Earth Sciences and Resources, China University of

Geosciences (Beijing), Beijing 100083, China.

1. Mounce, R. C. P. \& Wills, M. Phylogeny of Diania challenged. Nature 476, doi:10.1038/nature10266 (2011).

2. Legg, D. A. et al. Lobopodian phylogeny reanalysed. Nature 476, doi:10.1038/ nature10267 (2011).

3. Liu, J. et al. An armoured Cambrian lobopodian from China with arthropod-like appendages. Nature 470, 526-530 (2011).

4. Maddison, W. P. \& Maddison, D. R. MacClade: Analysis of Phylogeny and Character Evolution Version 3 (Sinauer Associates, 1992).

5. Swofford, D. L. PAUP*: Phylogenetic Analysis Using Parsimony (*and Other Methods) Version 4.0. (Sinauer Associates, 2002).

6. Faith, D. P. \& Cranston, P. S. Probability, parsimony, and Popper. Syst. Biol. 41, 252-257 (1992).

doi: $10.1038 /$ nature 10268 